\title{
Maternal Lopinavir/Ritonavir Is Associated with Fewer Adverse Events in Infants than Nelfinavir or Atazanavir
}

\author{
Christiana Smith, ${ }^{1}$ Adriana Weinberg, ${ }^{1,2,3}$ Jeri E. Forster, ${ }^{4}$ Myron J. Levin, ${ }^{1,2}$ Jill Davies, ${ }^{5,6}$ \\ Jennifer Pappas, ${ }^{7}$ Kay Kinzie, ${ }^{7}$ Emily Barr, ${ }^{1}$ Suzanne Paul, ${ }^{1}$ and Elizabeth J. McFarland ${ }^{1}$ \\ ${ }^{1}$ Division of Infectious Diseases, Department of Pediatrics, University of Colorado School of Medicine and Children's Hospital Colorado, \\ Aurora, CO 80045, USA \\ ${ }^{2}$ Department of Medicine, University of Colorado School of Medicine, Aurora, CO 80045, USA \\ ${ }^{3}$ Department of Pathology, University of Colorado School of Medicine, Aurora, CO 80045, USA \\ ${ }^{4}$ Department of Biostatistics and Informatics, Colorado School of Public Health, Aurora, CO 80045, USA \\ ${ }^{5}$ Department of Obstetrics and Gynecology, University of Colorado School of Medicine, Aurora, CO 80045, USA \\ ${ }^{6}$ Denver Health Medical Center, Denver, CO 80204, USA \\ ${ }^{7}$ Children's Hospital Colorado, Aurora, CO 80045, USA
}

Correspondence should be addressed to Christiana Smith; christiana.smith@childrenscolorado.org

Received 18 December 2015; Accepted 9 March 2016

Academic Editor: Bryan Larsen

Copyright (C) 2016 Christiana Smith et al. This is an open access article distributed under the Creative Commons Attribution License, which permits unrestricted use, distribution, and reproduction in any medium, provided the original work is properly cited.

\begin{abstract}
Combination antiretroviral therapy (cART) is successfully used for prevention of perinatal HIV transmission. To investigate safety, we compared adverse events (AE) among infants exposed to different maternal cART regimens. We reviewed 158 HIV-uninfected infants born between 1997 and 2009, using logistic regression to model grade $\geq 1 \mathrm{AE}$ and grade $\geq 3 \mathrm{AE}$ as a function of maternal cART and confounding variables (preterm, C-section, illicit drug use, race, ethnicity, infant antiretrovirals, and maternal viremia). Frequently used cART regimens included zidovudine (63\%), lamivudine (80\%), ritonavir-boosted lopinavir (37\%), nelfinavir (26\%), and atazanavir (10\%). At birth, anemia occurred in 13/140 infants (9\%), neutropenia in 27/107 (25\%), thrombocytopenia in 5/133 (4\%), and liver enzyme elevation in 21/130 (16\%). Corresponding rates of AE at 4 weeks were 59/141 (42\%), 54/130 (42\%), 3/137 (2\%), and 3/104 (3\%), respectively. Serious AE (grade $\geq 3$ ) exceeded $2 \%$ only for neutropenia (13\% at birth; $9 \%$ at 4 weeks). Compared with infants exposed to maternal lopinavir/ritonavir, infants exposed to nelfinavir and atazanavir had a 5-fold and 4-fold higher incidence of $\mathrm{AE}$ at birth, respectively. In conclusion, hematologic and hepatic AE were frequent, but rarely serious. In this predominantly protease inhibitor-treated population, lopinavir/ritonavir was associated with the lowest rate of infant AE.
\end{abstract}

\section{Introduction}

The prevention of perinatal transmission of HIV is one of the most successful public health interventions of the last few decades. The use of maternal combination antiretroviral therapy (cART), when combined with infant postnatal prophylaxis, has reduced transmission rates to less than $1 \%$ in developed countries $[1,2]$. This is a remarkable achievement, but concerns remain regarding toxicity in these infants after exposure to multiple antiretrovirals (ARV) in utero, during delivery, and in early infancy [3].

Spontaneous preterm delivery and low birth weight have been associated with HIV infection during pregnancy.
Although not a uniform finding across studies, the use of protease inhibitors (PI) during pregnancy may increase the incidence of preterm delivery [4-6]. Mitochondrial and nuclear genotoxicity are associated with in utero exposure to nucleoside reverse transcriptase inhibitors (NRTI) [7, 8]. In addition, laboratory adverse events (AE) have been described in ARV-exposed infants, including hematologic cytopenias and disruption of liver function. The NRTI are known to alter in vitro hematopoiesis $[9,10]$. Transient macrocytic anemia was the most common side effect in infants exposed to zidovudine (ZDV) pre- and postnatally in the landmark PACTG 076 study [11, 12], and several reports describe profound neonatal anemia after ARV exposure in 
utero $[13,14]$. Other studies have confirmed the link between ARV exposure and neonatal neutropenia, lymphopenia, and thrombocytopenia [15-20]. Liver dysfunction is described less frequently, although several studies have demonstrated elevated neonatal aspartate aminotransferase (AST), alanine aminotransferase (ALT), and bilirubin after exposure to perinatal ARV [21-25]. Increasing complexity of maternal cART correlates with an increased risk for hematologic and hepatic AE [15, 17, 21, 22, 26-28].

As new ARV and more complex cART regimens are administered for the prevention of perinatal HIV transmission, it is important to identify the toxicities associated with both established and novel regimens in order to inform best choices [29-31]. Prior studies have compared infant AE after exposure to different classes of ARV, for example, nonnucleoside reverse transcriptase inhibitor- (NNRTI-) based cART and PI-based cART $[22,27]$. In this study, we examine infant AE after exposure to different maternal drugs within ARV classes.

\section{Materials and Methods}

2.1. Study Design. This study was approved by the Colorado Multiple Institutional Review Board and exempted from informed consent. This was a retrospective chart review of 190 pregnancies complicated by HIV infection that were managed by the Children's Hospital Immunodeficiency Program (CHIP) in Denver. CHIP is the reference center for the care of HIV-infected pregnant women in Colorado and neighboring states. Data were abstracted for all pregnancies from 1997 to 2009 that resulted in a live infant birth. Maternal data collected included demographics, ARV use, illicit drug use, mode of delivery, hematologic and hepatic laboratory values, CD4 count, and viral load. Undetectable viral load was defined as $<400$ copies $/ \mathrm{mL}$, as this was the lower limit of detection for the earliest data. Duration of maternal viremia during pregnancy was defined as the number of days from the estimated conception date until either the date of the first undetectable viral load measurement after which all subsequent viral load measurements were undetectable or, if there was no sustained viral suppression, the number of days between the estimated conception date and the date of infant birth. The estimated conception date was calculated from infant gestational age determined at birth. Infant data collected included gestational age (preterm defined as <37 weeks), birth weight (small for gestational age (SGA) defined as $<3$ rd percentile of expected weight for gestational age), postnatal ARV prophylaxis, laboratory values (hemoglobin, neutrophil count, platelets, AST, ALT, and total bilirubin), and hospitalizations or illnesses. Infant complete blood count and liver function panel was assessed at birth (age 0-7 days) and at 4 weeks ( \pm 2 weeks). Laboratory toxicities were graded using the Division of AIDS Table for Grading the Severity of Adult and Pediatric Adverse Events [32]. If the medical record did not specify an upper limit of normal for AST and ALT, the limits imposed were $60 \mathrm{IU} / \mathrm{L}$ and $65 \mathrm{IU} / \mathrm{L}$ for AST and ALT, respectively, as these are the limits used by the Children's Hospital Colorado Laboratory. Infant infection status was monitored using HIV RNA and/or DNA PCR at birth, 2 weeks, 4 weeks, 6 weeks, and 4 months of age and HIV antibody testing starting at 12 months of age and repeated every 3-6 months until seroreversion was demonstrated.

2.2. Prophylaxis Regimens. Pregnant women received a clinician-prescribed antenatal ARV regimen that consisted of cART ( $\geq 3$ ARV from $\geq 2$ ARV classes) in most cases; modifications during the pregnancy were based on viral genotype, virologic response, safety, and tolerability, as previously described [33-35]. PI serum levels were routinely monitored and doses adjusted to achieve a trough above the 25th percentile for nonpregnant adults. Infant ARV exposure was assigned based on the maternal ARV received for $\geq 28$ days during the 35 days immediately prior to delivery, thereby setting a minimum period of in utero drug exposure occurring near the time of infant laboratory assessment. Other maternal ARV received before the 35 days immediately prior to delivery was not accounted for in the analysis.

Most infants were prescribed postnatal prophylaxis consisting of six weeks of ZDV. In some infants, ZDV was replaced by stavudine ( $\mathrm{d} 4 \mathrm{~T})$ due to anemia, neutropenia, or both. In situations where the risk of perinatal transmission was increased, infants received two- or three-drug ARV. A detailed description of the infant postnatal prophylaxis prescribed and associated adverse events has been previously reported [36].

2.3. Statistical Analysis. The analysis included infants with at least one laboratory value at either birth or 4 weeks. The statistical analyses require that all observations (infants) are independent of one another or the results may be biased. Given that twins have identical exposure in utero and in order not to underestimate associations between $\mathrm{AE}$ and ARV, the twin with the lower grade toxicity was excluded from each pair. Maternal and infant characteristics were compared using $t$-tests, chi-square tests, and Fisher's exact tests, as appropriate. Because most infants were exposed to an antenatal ARV regimen containing two NRTI and a PI, AE were compared between infants exposed to different NRTI and between infants exposed to different PI. The number of infants exposed to nevirapine $(n=10)$ was too small for comparison. Rates of preterm birth and SGA were compared as a univariate analysis. Logistic regression was used to model grade $\geq 1$ laboratory $\mathrm{AE}$ and grade $\geq 3$ laboratory $\mathrm{AE}$ (yes/no) as a function of maternal ARV received for $\geq 28$ days during the 35 days immediately prior to delivery and any confounding variable that changed the odds ratio $(\mathrm{OR}$, $95 \%$ confidence interval) by $>20 \%$. Confounding variables used in the multivariate analyses include preterm birth, infant exposure to ZDV monoprophylaxis versus combination prophylaxis, mechanism of delivery, maternal race and ethnicity, maternal illicit drug use, and duration of maternal viremia during the pregnancy. Statistical significance was defined by $p \leq 0.05$.

\section{Results}

3.1. Maternal Characteristics and Antiretroviral Regimens. One hundred and sixty-five mothers were managed at CHIP 
TABLE 1: Infant demographics and select obstetrical characteristics.

\begin{tabular}{|c|c|}
\hline Characteristic & Number of infants/number evaluated $(\%)^{\mathrm{b}}$ \\
\hline \multicolumn{2}{|l|}{ Race $^{c}$} \\
\hline Caucasian & $92 / 158(58 \%)$ \\
\hline African American & $53 / 158(34 \%)$ \\
\hline American Indian/Alaskan Native & $2 / 158(1 \%)$ \\
\hline Other/unknown & $11 / 158(7 \%)$ \\
\hline \multicolumn{2}{|l|}{ Ethnicity $^{c}$} \\
\hline Hispanic & $55 / 158(34 \%)$ \\
\hline Not Hispanic & $94 / 158(60 \%)$ \\
\hline Other/unknown & $10 / 158(6 \%)$ \\
\hline Sex, male & $81 / 152(53 \%)$ \\
\hline \multicolumn{2}{|l|}{ Entry maternal CD 4 count $^{\mathrm{d}}$} \\
\hline$<200$ cells $/ \mathrm{mm}^{3}$ & $7 / 101(7 \%)$ \\
\hline $200-500$ cells $/ \mathrm{mm}^{3}$ & $40 / 101(40 \%)$ \\
\hline$>500$ cells $/ \mathrm{mm}^{3}$ & $54 / 101(53 \%)$ \\
\hline Entry median maternal HIV RNA copies $/ \mathrm{mL}$ in plasma ${ }^{\mathrm{d}}$ (range) $N=109$ & $2120(<20-213,191)$ \\
\hline Antiretrovirals initiated before conception & $44 / 152(29 \%)$ \\
\hline Maternal illicit drug use & $19 / 158(12 \%)$ \\
\hline Mean gestational age in weeks (range) $N=150$ & $37.7(25-41.7)$ \\
\hline Preterm deliveries & $26 / 150(17 \%)$ \\
\hline Deliveries via Cesarean section & $64 / 158(41 \%)$ \\
\hline
\end{tabular}

${ }^{a}$ Denominator represents the number of infants with available data.

${ }^{\mathrm{b}}$ Unless units of measurement are otherwise indicated.

${ }^{c}$ Infant race and ethnicity determined by maternal self-report.

${ }^{\mathrm{d}}$ From earliest known maternal laboratory values during pregnancy.

for 190 pregnancies, resulting in 196 live births. Of these, 163 infants (5 twins) had at least one laboratory value available for analysis. The analysis cohort consisted of 158 mother/infant pairs after excluding one infant from each twin pair. There were no perinatal transmissions of HIV. The infant demographics and maternal HIV and obstetrical characteristics are reported in Table 1. Severe maternal immune suppression was rare; only $7 \%$ of women had a CD 4 count $<200$ cells $/ \mathrm{mm}^{3}$ in early pregnancy. One hundred twenty-seven women $(80 \%)$ received CART for at least 28 days prior to delivery. Fifteen women received cART for less than 28 days prior to delivery. Sixteen women received a less intensive ARV regimen. Maternal ARV received in the last month of pregnancy are detailed in Table 2. PI-based cART was received by $77 \%$ of women, including lopinavir with ritonavir (LPV/r, 37\%), nelfinavir (NFV, 26\%), atazanavir (ATV, 10\%, including $2 \%$ without ritonavir), and saquinavir (4\%). The majority of women received ZDV (63\%), but $13 \%, 9.5 \%$, and $4 \%$ received $\mathrm{d} 4 \mathrm{~T}$, tenofovir (TDF), and abacavir $(\mathrm{ABC})$, respectively. There were few differences in maternal and infant characteristics between the two largest treatment groups, LPV/r versus NFV (Supplementary Table 1 in the Supplementary Material available online at http://dx.doi.org/10.1155/2016/9848041).

3.2. Overall Frequency of Adverse Events. Laboratory AE in the first four weeks of life were common, with three quarters of infants having an $\mathrm{AE}$ (any grade) at either birth or 4 weeks. The frequency of an $\mathrm{AE}$ grade $\geq 3$ in any laboratory category was $12 \%$ at birth and $16 \%$ at 4 weeks. Neutropenia was most common, with $25 \%$ and $42 \%$ of infants having any grade AE, and $13 \%$ and $9 \%$ having grade $\geq 3 \mathrm{AE}$, at birth and 4 weeks, respectively (Figure 1). Anemia was the next most common, with $9 \%$ and $42 \%$ of infants having any grade AE, but only $1 \%$ and $0 \%$ having grade $\geq 3 \mathrm{AE}$, at birth and 4 weeks, respectively. Rates of AST AE fell from $16 \%$ at birth to 3\% at 4 weeks. Rates of ALT AE and thrombocytopenia were low at birth and at 4 weeks. No infants had hyperbilirubinemia at birth.

The 26 (17\%) infants born preterm were more likely than term infants to develop an $\mathrm{AE}$ of any grade (93\% versus $71 \%$, $p=0.03)$ or grade $\geq 3$ ( $46 \%$ versus $17 \%, p=0.003)$ at either birth or 4 weeks. Four preterm infants required blood transfusions due to severe anemia. No infants suffered a serious bacterial infection or a bleeding disorder as a complication of neutropenia or thrombocytopenia, and no infants developed liver failure as a result of hepatic inflammation. No infants developed renal or cardiac dysfunction.

3.3. Association of Maternal ARV with Infant Adverse Events. Rates of infant laboratory AE relative to antenatal exposure to maternal ARV were compared in a multivariate analysis. At birth, infants exposed to maternal NFV compared to $\mathrm{LPV} / \mathrm{r}$ had a higher rate of anemia (OR 7.4 (95\% CI 1.439.0), $p=0.02$ ), neutropenia (OR 10.6 (95\% CI 1.7-66.4), $p=0.01$ ), and any AE (OR $5.3(95 \%$ CI 1.9-14.9), $p=0.002$ ) (Figure 2). This association was maintained when limited to infants exposed to the same maternal NRTI backbone (ZDV 
TABLE 2: Antenatal antiretroviral exposure of infants.

\begin{tabular}{lc}
\hline Drug $^{\mathrm{a}}$ & $\begin{array}{c}\text { Number exposed (\%) } \\
N=158\end{array}$ \\
\hline Nucleoside reverse transcriptase inhibitors & $7(4 \%)$ \\
$\quad$ Abacavir & $15(9.5 \%)$ \\
Emtricitabine & $126(80 \%)$ \\
Lamivudine & $21(13 \%)$ \\
Stavudine & $15(9.5 \%)$ \\
Tenofovir & $99(63 \%)$ \\
Zidovudine & \\
Nonnucleoside reverse transcriptase inhibitors & $10(6 \%)$ \\
$\quad$ Nevirapine & $16(10 \%)$ \\
Protease inhibitors & $59(37 \%)$ \\
Atazanavir \pm ritonavir & \\
Lopinavir + ritonavir & $41(26 \%)$ \\
Nelfinavir & $7(4 \%)$ \\
Saquinavir \pm ritonavir & \\
\hline
\end{tabular}

${ }^{a}$ Maternal treatment administered for $\geq 28$ days of the 35 days immediately preceding delivery.

${ }^{b}$ Thirteen of sixteen women received ritonavir-boosted atazanavir.

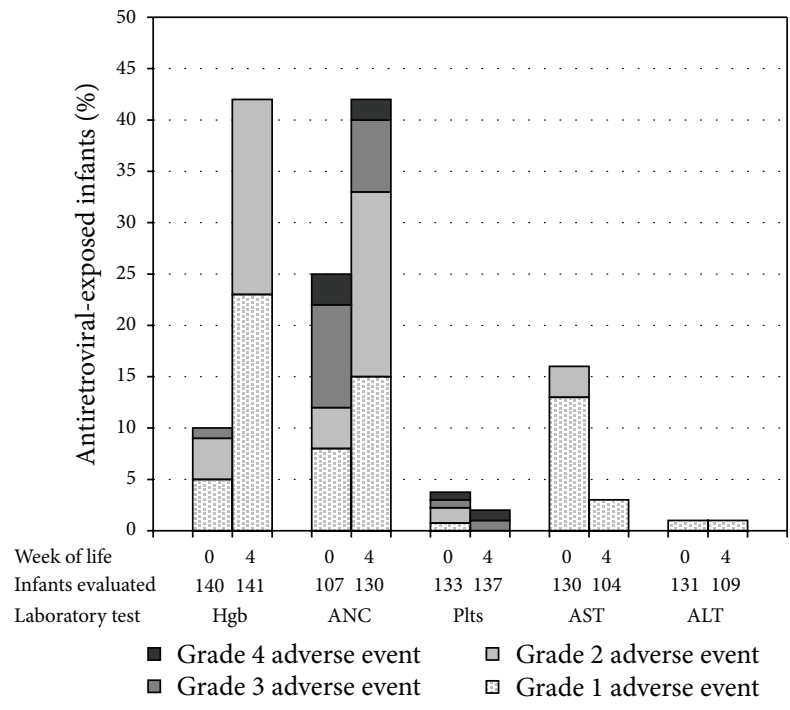

FIGURE 1: Frequency of infant adverse events. Bars represent percentages of infants with laboratory adverse events at birth (age 0-7 days) and at 4 weeks ( \pm 2 weeks). Hgb, hemoglobin; ANC, absolute neutrophil count; plts, platelet count; AST, aspartate aminotransferase; ALT, alanine aminotransferase.

and lamivudine) (Table 3). The difference was primarily the result of grade $1 \mathrm{AE}$; rates of grade $\geq 3 \mathrm{AE}$ remained relatively low in both groups (9\% versus $14 \%$ of LPV/r-exposed versus NFV-exposed infants, resp.; Figure 2). Exposure to maternal ATV was also associated with a higher rate of any laboratory $\mathrm{AE}$ compared to $\mathrm{LPV} / \mathrm{r}$ (OR 4.2 (95\% CI 1.0-17.5), $p=$ $0.046)$ with the difference primarily due to higher rates of neutropenia (OR 18.3 (95\% CI 1.2-267.8), $p=0.03$ ) (Figure 2). At 4 weeks, there were no longer significant differences in $\mathrm{AE}$ between infants exposed to different PI (Table 4).

Additional maternal and infant characteristics were examined to determine whether variables not included in the multivariate analysis might contribute to differences in infant $\mathrm{AE}$ at birth for the NFV and LPV/r groups, which had a sample size large enough for analysis (Supplementary Table 1). The mean estimated duration of maternal viremia was longer in the NFV-exposed group than in the LPV/rexposed group (172 versus 125 days, $p=0.05$ ). However, the association of infant AE with NFV versus LPV/r exposure remained significant when duration of maternal viremia was included as a covariate in the multivariate analysis (OR 7.9 (95\% CI 2.4-26.2), $p=0.0007$ ). In addition, a univariate analysis demonstrated that the duration of maternal viremia did not correlate with the frequency of infant AE (OR 1.0 (95\% CI 0.99-1.0), $p=0.57$ ). An anticipated difference between the treatment groups was the year of delivery. NFV was prescribed in an earlier time period (1997-2007); LPV/r was first prescribed in 2003 and surpassed NFV as the most commonly used PI at our center by 2004 . Other variables did not differ including the duration of antenatal exposure to NFV or LPV/r or the proportion of women who received the PI prior to conception (Supplementary Table 1).

Most women were treated with ZDV, but a substantial number received $\mathrm{ABC}, \mathrm{d} 4 \mathrm{~T}$, or TDF; therefore, rates of infant $\mathrm{AE}$ were compared between these groups. There were no significant differences at birth in rates of anemia, neutropenia, thrombocytopenia, or liver enzyme elevation between infants exposed to maternal ZDV compared with $\mathrm{ABC}$, d4T, or TDF (Supplementary Table 2).

Rates of preterm birth and SGA for infants exposed to different maternal PI were compared in a univariate analysis. No significant differences were found in rates of preterm birth (5/16 (31\%), 9/58 (16\%), and 5/37 (14\%), $p=0.26$ ) 
TABLE 3: Infant adverse events at birth associated with maternal lopinavir/ritonavir versus nelfinavir, in combination with zidovudine and lamivudine ${ }^{\mathrm{a}}$.

\begin{tabular}{|c|c|c|c|}
\hline Laboratory test ${ }^{\mathrm{b}}$ & Lopinavir/ritonavir ${ }^{\mathrm{c}}$ & Nelfinavir ${ }^{c}$ & $\begin{array}{c}\text { Odds ratio }(95 \% \mathrm{CI}) \\
p \text { value }\end{array}$ \\
\hline $\mathrm{Hgb}$ & $2 / 34(6 \%)$ & $6 / 29(21 \%)$ & $\begin{array}{c}6.3(1.01-39.8) \\
p=0.049\end{array}$ \\
\hline ANC & $2 / 22(9 \%)$ & $7 / 25(28 \%)$ & $\begin{array}{c}\mathrm{N} / \mathrm{A}^{\mathrm{d}} \\
p=0.14\end{array}$ \\
\hline AST & $4 / 34(12 \%)$ & $7 / 25(28 \%)$ & $\begin{array}{c}3.5(0.73-16.7) \\
\quad p=0.12\end{array}$ \\
\hline Highest grade AE, all tests & $10 / 35(29 \%)$ & $17 / 29(59 \%)$ & $\begin{array}{c}4.9(1.6-15.4) \\
p=0.006\end{array}$ \\
\hline
\end{tabular}

Hgb, hemoglobin; ANC, absolute neutrophil count; AST, aspartate aminotransferase.

${ }^{a}$ Multivariate analysis using logistic regression was used to model grade $\geq 1 \mathrm{AE}$ (yes/no) as a function of maternal antiretroviral treatment. Groups restricted to infants born to mothers treated with zidovudine/lamivudine in combination with either lopinavir/ritonavir or nelfinavir.

${ }^{\mathrm{b}}$ There were no adverse events for bilirubin, 1 adverse event for alanine aminotransferase in the lopinavir/ritonavir group, and 3 adverse events for platelet count in the nelfinavir group, not shown separately but included in the maximum adverse events.

${ }^{c}$ Number of infants with adverse event/number of infants exposed (\%).

${ }^{\mathrm{d}}$ Fisher's exact test reported.

TABLE 4: Infant adverse events at 4 weeks associated with exposure to maternal lopinavir/ritonavir versus nelfinavir or atazanavir . $^{\mathrm{a}}$

\begin{tabular}{|c|c|c|c|c|c|}
\hline Laboratory test $^{\mathrm{b}}$ & Lopinavir/ritonavir ${ }^{c}$ & Nelfinavir ${ }^{c}$ & $\begin{array}{c}\text { Odds ratio }(95 \% \mathrm{CI}) \\
\quad p \text { value }^{\mathrm{d}} \\
\end{array}$ & Atazanavir ${ }^{\mathrm{c}}$ & $\begin{array}{c}\text { Odds ratio }(95 \% \mathrm{CI}) \\
p \text { value } \\
\end{array}$ \\
\hline $\mathrm{Hgb}$ & 19/53 (36\%) & $14 / 34(41 \%)$ & $\begin{array}{c}1.3(0.52-3.0) \\
p=0.62\end{array}$ & 6/14 (43\%) & $\begin{array}{c}1.3(0.41-4.4) \\
p=0.63\end{array}$ \\
\hline ANC & $24 / 48(50 \%)$ & $11 / 30(37 \%)$ & $\begin{array}{c}0.58(0.23-1.5) \\
p=0.25\end{array}$ & $5 / 14(36 \%)$ & $\begin{array}{c}0.56(0.16-1.9) \\
p=0.35\end{array}$ \\
\hline Bili & $11 / 52(21 \%)$ & 9/26 (35\%) & $\begin{array}{c}2.5(0.75-8.1) \\
p=0.14\end{array}$ & $3 / 7(43 \%)$ & $\begin{array}{c}2.0(0.35-11.4) \\
p=0.44\end{array}$ \\
\hline Highest grade AE, all tests & $38 / 55(69 \%)$ & $22 / 35(63 \%)$ & $\begin{array}{c}0.76(0.31-1.8) \\
p=0.54\end{array}$ & 9/14 (64\%) & $\begin{array}{c}0.62(0.16-2.4) \\
p=0.49\end{array}$ \\
\hline
\end{tabular}

Hgb, hemoglobin; ANC, absolute neutrophil count; Bili, total bilirubin; AE, adverse event.

${ }^{a}$ Multivariate analysis using logistic regression was used to model grade $\geq 1 \mathrm{AE}$ (yes/no) as a function of maternal antiretroviral treatment. No significant differences were found.

${ }^{\mathrm{b}}$ There were no adverse events for alanine aminotransferase, 1 adverse event each for aspartate aminotransferase in the lopinavir/ritonavir and atazanavir groups, and 1 adverse event for platelet count in each group, not shown separately but included in the maximum adverse events.

${ }^{c}$ Number of adverse events/number exposed (\%).

${ }^{\mathrm{d}}$ Lopinavir/ritonavir versus nelfinavir.

${ }^{\mathrm{e}}$ Lopinavir/ritonavir versus atazanavir.

or SGA $(2 / 16(13 \%), 7 / 56(13 \%)$, and $4 / 35(11 \%), p>$ 0.99 ) between infants exposed to ATV, LPV/r, and NFV, respectively, although the small sample size limited the power to detect a difference.

\section{Discussion}

Low-grade laboratory AE were common at birth and 4 weeks in the cART-exposed infants, but grade $\geq 3 \mathrm{AE}$ were rare. Anemia and neutropenia made up the majority of $\mathrm{AE}$, and neutropenia comprised nearly all grade $\geq 3 \mathrm{AE}$. Our results are in agreement with those of previous studies, which showed frequent, but low-grade, hematologic abnormalities among ARV-exposed infants. Pacheco et al. reported significantly decreased infant hemoglobin and neutrophil values, and Mussi-Pinhata et al. described anemia at hospital discharge in $24 \%$ of ARV-exposed infants, although in both studies nearly all AE were of grade 1 or $2[17,23]$. Read et al. described grade $\geq 3$ anemia or neutropenia in less than $10 \%$ of ARV-exposed infants in the first six weeks of life [19]. Four infants in our study required a blood transfusion due to anemia, all of whom were born at $\leq 32$ weeks of gestation. Preterm infants are likely to require blood transfusion even when unexposed to ARV, with about $80 \%$ of US infants with birth weight $<1500$ grams requiring at least one transfusion [37]. None of the infants born $\geq 37$ weeks of gestation in this study developed clinically significant AE. Although the majority of AE in these HIVand ARV-exposed infants were of low grade, the mechanism underlying the AE is not fully understood and the long term effects of these early toxicities are unknown.

We found increased infant laboratory AE at birth associated with exposure to maternal NFV and ATV compared with $\mathrm{LPV} / \mathrm{r}$. The difference was observed even when the analysis was restricted to mother/infant pairs with the same NRTI background. At 4 weeks, no difference was detectable between these groups, suggesting that the effect is either 


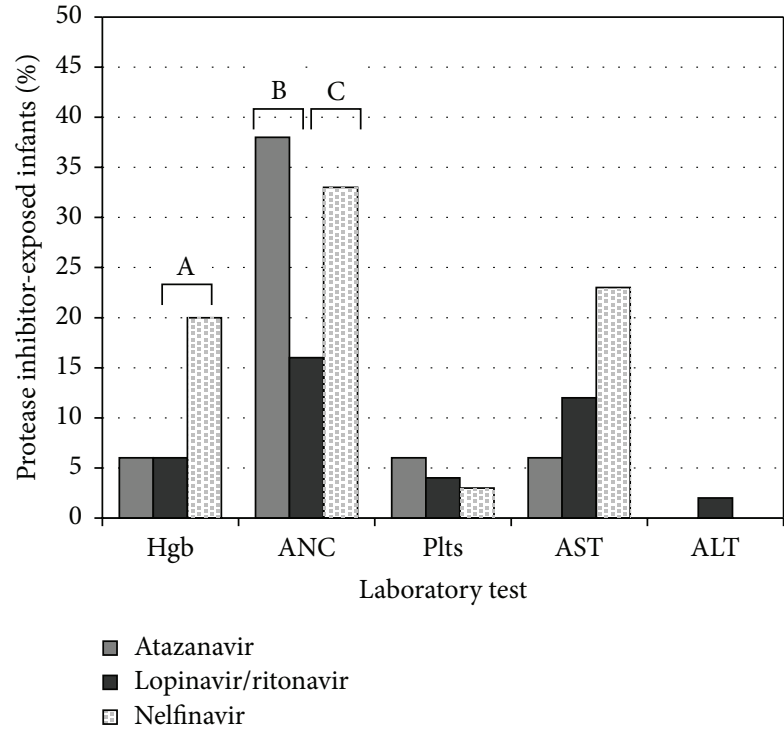

(a)

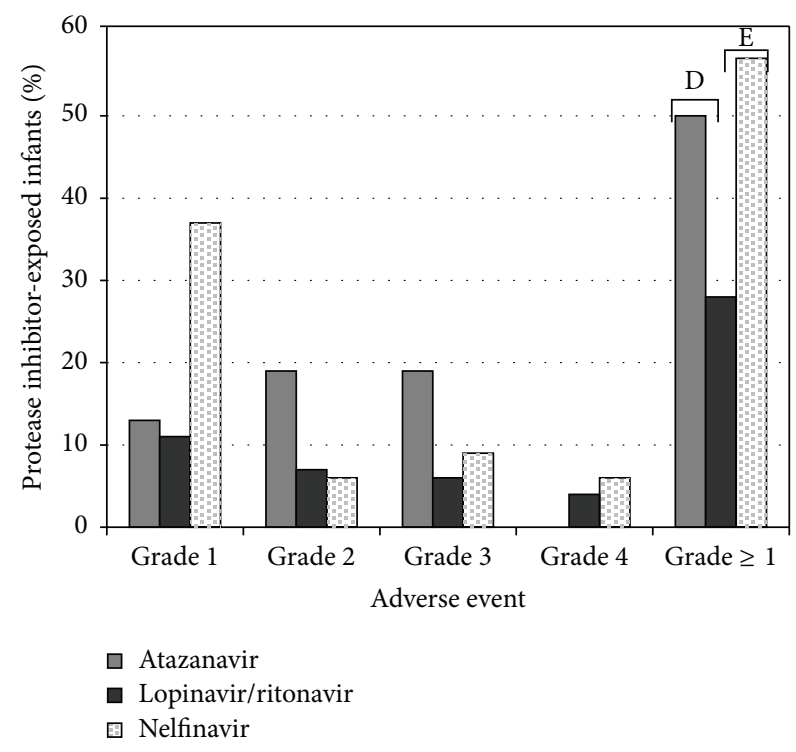

(b)

Figure 2: Frequency of infant adverse events at birth by maternal protease inhibitor. Bars represent the percentage of infants with a laboratory adverse event of any grade (a) or the percentage of infants whose most severe adverse event was represented by that particular grade (b). Number of infants per group: atazanavir \pm ritonavir (16), lopinavir/ritonavir (54), and nelfinavir (35). Hgb, hemoglobin; ANC, absolute neutrophil count; plts, platelet count; AST, aspartate aminotransferase; ALT, alanine aminotransferase. ${ }^{\mathrm{A}} \mathrm{OR}=7.4(95 \% \mathrm{CI} 1.4-39.0), p=0.02$; ${ }^{\mathrm{B}} \mathrm{OR}=18.3(95 \% \mathrm{CI} 1.2-267.8), p=0.03 ;{ }^{\mathrm{C}} \mathrm{OR}=10.6(95 \% \mathrm{CI} 1.7-66.4), p=0.01 ;{ }^{\mathrm{D}} \mathrm{OR}=4.2(95 \% \mathrm{CI} 1.0-17.5), p=0.046 ;{ }^{\mathrm{E}} \mathrm{OR}=5.3(95 \%$ CI 1.9-14.9), $p=0.002$.

transient or masked by the effect of postnatal ARV. Our multivariate analysis controlled for exposure to postnatal ZDV monoprophylaxis versus combination prophylaxis. The association of laboratory AE with particular antenatal ARV within the PI class is reported here for the first time. Of note, Bellon Cano et al. described increased anemia, thrombocytopenia, and liver enzyme elevation after exposure to PI-based cART (of which most regimens contained NFV) compared with two or three NRTI or NNRTI-based cART [22].

The mechanism of the PI effect on hematologic AE is unclear. Fetal hematopoiesis may be more susceptible to the effects of PI, as it occurs mainly in the liver and peripheral lymphoid tissues. PI cross the placenta to some degree; albeit due to their large molecular weight and high protein binding capacity, they do not reach therapeutic levels in cord blood. Cord blood to maternal plasma ratios for ATV, NFV, and LPV range between $0.13-0.24,0.16-0.22$, and $0-0.49$, respectively [38]. Amniotic fluid to maternal plasma ratios have been described for NFV (0.14-0.44) and LPV (0.08) $[39,40]$. Although serum levels for some PI may be low during pregnancy [41], in this cohort plasma PI levels were routinely monitored during pregnancy and doses adjusted to achieve a trough above the 25th percentile for nonpregnant adults. Therefore, the difference in infant $\mathrm{AE}$ is not likely attributable to differences in maternal PI drug levels.

Because this was a retrospective study, it is possible that the maternal receipt of various PI could be a marker for another confounding variable that contributed to infant AE. Examination for potential confounding variables between the $\mathrm{NFV}$ and LPV/r groups identified that on average women receiving NFV had a longer duration of viremia than women receiving LPV/r. However, viremia did not correlate with increased risk of infant $\mathrm{AE}$, and inclusion of maternal viremia as a covariate did not alter the relationship of infant $\mathrm{AE}$ with exposure to NFV versus LPV/r.

Owing to its limited antiviral activity, NFV is no longer recommended for use in pregnant women [42]. However, there may be rare clinical scenarios in which NFV use might be considered, such as for patients who cannot tolerate or have resistance to other ARV that are included in the preferred and alternative regimens for pregnancy. The results of this study may be further rationale to avoid NFV during pregnancy.

We found that ATV exposure was associated with a higher incidence of neutropenia compared with LPV/r. However, infants in this study who were exposed to ATV did not show increased rates of liver enzyme elevation or hyperbilirubinemia compared with infants exposed to other PI. These findings are in contrast to those of Mandelbrot et al. who described a series of twenty-three infants exposed in utero to ATV, nine of whom developed elevated serum bilirubin concentrations at birth and five required phototherapy in the first three days of life [25]. Notably, a subsequent study found no association between ATV exposure and neonatal hyperbilirubinemia [43]. Our data need to be further confirmed in larger studies, because they were derived from only sixteen infants exposed to ATV, resulting in an OR with relatively large confidence intervals.

Although ZDV is commonly associated with hematologic side effects in exposed infants, we did not find more hematologic $\mathrm{AE}$ at birth among infants exposed to antenatal 
ZDV compared with those exposed to ABC, d4T, or TDF, but the number of infants exposed to the alternative NRTI was small. Few studies compare infant toxicities after in utero exposure to different NRTI, although Vivanti et al. demonstrated decreased genotoxicity in cord blood of infants exposed in utero to TDF versus ZDV [44].

Preterm birth has been described previously in association with in utero exposure to PI [4-6]. We did not find an association of preterm birth or SGA with LPV/r, NFV, or ATV, but the power to detect differences was limited by our small sample size. In addition, data describing other risk factors for preterm birth or SGA were not available in our data set. Perry et al. recently showed no differences in preterm birth or birth weight in infants exposed to maternal $\mathrm{LPV} / \mathrm{r}$ versus ATV; this study did not report infant laboratory outcomes [45].

We acknowledge several limitations in this study. The sample size was relatively small. Mothers and infants were not randomized to various ARV in our study; they received a regimen that was clinically appropriate based on severity of disease, tolerability, viral response to therapy, and risk of perinatal HIV transmission. Although two groups of infants for whom we found a significant difference in AE (those exposed to $\mathrm{LPV} / \mathrm{r}$ and NFV) were cared for in different time periods, we also found differences between the effect of LPV/r and ATV exposure, whose use was roughly contemporaneous. Despite these limitations, this study permits comparison of infant AE within classes of ARV, whereas most similar studies have compared between classes of ARV. It also provides data on newer ARV used for prevention of perinatal transmission.

\section{Conclusions}

In summary, when used in maternal cART for prevention of perinatal transmission of HIV, NFV and ATV were associated with increased infant AE compared to LPV/r. As ATV use in pregnant women is increasing, larger, preferably randomized studies of infants exposed to LPV/r versus ATV/r are needed to evaluate laboratory $\mathrm{AE}$ and to clarify any association with prematurity. Investigations into the safest maternal ARV regimens and timing of $\mathrm{ARV}$ initiation are important for optimizing outcomes for HIV-exposed infants, especially those with higher risk of hematologic and hepatic toxicity, such as preterm infants and infants in resource-limited settings.

\section{Disclosure}

These data were presented in part at the 49th annual meeting of the Infectious Diseases Society of America (poster presentation), Boston, MA, October 2011.

\section{Competing Interests}

This work was partially supported by funding from Colorado HIV-1 Research Training Program Fellowship Position (Grant T32AI007447-23) and by contributions from Pfizer (Project no. 2580628) and Abbott (Project no. 2580614). Dr. Weinberg receives research funds from MedImmune,
Becton Dickinson, Roche, Cepheid, Diagnostic Hybrids, and Sanofi Pasteur. Dr. Levin receives research funds, fees for consultation, and royalties from Patent no. 5,997,880, entitled "Method for Alleviating Varicella Related PostHerpetic Neuralgia” from Merck \& Co. Dr. Levin also receives research funds from and sits on the adjudication committee for GlaxoSmithKline. Dr. McFarland receives research funds from Gilead. In addition, Drs. Levin and Weinberg share competing interests due to their marital association. For the remaining authors, none were declared.

\section{Acknowledgments}

The authors would like to thank Carol Salbenblatt and Annie Vasquez for their assistance with data entry and Jessica Peterson for her assistance with record retrieval.

\section{References}

[1] J. C. Forbes, A. M. Alimenti, J. Singer et al., "A national review of vertical HIV transmission," AIDS, vol. 26, no. 6, pp. 757-763, 2012.

[2] L. Navér, S. Lindgren, E. Belfrage et al., "Children born to HIV1-infected women in Sweden in 1982-2003: trends in epidemiology and vertical transmission," Journal of Acquired Immune Deficiency Syndromes, vol. 42, no. 4, pp. 484-489, 2006.

[3] L. M. Mofenson, "Editorial commentary: new challenges in the elimination of pediatric HIV infection: the expanding population of HIV-exposed but uninfected children," Clinical Infectious Diseases, vol. 60, no. 9, pp. 1357-1360, 2015.

[4] D. H. Watts, P. L. Williams, D. Kacanek et al., "Combination antiretroviral use and preterm birth," The Journal of Infectious Diseases, vol. 207, no. 4, pp. 612-621, 2013.

[5] European Collaborative Study, "Exposure to antiretroviral therapy in utero or early life: the health of uninfected children born to HIV-infected women," Journal of Acquired Immune Deficiency Syndromes, vol. 32, no. 4, pp. 380-387, 2003.

[6] J. Sibiude, J. Warszawski, R. Tubiana et al., "Premature delivery in HIV-infected women starting protease inhibitor therapy during pregnancy: role of the ritonavir boost?" Clinical Infectious Diseases, vol. 54, no. 9, pp. 1348-1360, 2012.

[7] I. André-Schmutz, L. Dal-Cortivo, E. Six et al., "Genotoxic signature in cord blood cells of newborns exposed in utero to a zidovudine-based antiretroviral combination," Journal of Infectious Diseases, vol. 208, no. 2, pp. 235-243, 2013.

[8] S. Blanche, M. Tardieu, V. Benhammou, J. Warszawski, and P. Rustin, "Mitochondrial dysfunction following perinatal exposure to nucleoside analogues," AIDS, vol. 20, no. 13, pp. 16851690, 2006.

[9] N. Dainiak, M. Worthington, M. A. Riordan, S. Kreczko, and L. Goldman, “ 3 '-Azido-3'-deoxythymidine (AZT) inhibits proliferation in vitro of human haematopoietic progenitor cells," British Journal of Haematology, vol. 69, no. 3, pp. 299-304, 1988.

[10] M. M. Shah, Y. Li, and R. D. Christensen, "Effects of perinatal zidovudine on hematopoiesis: a comparison of effects on progenitors from human fetuses versus mothers," AIDS, vol. 10, no. 11, pp. 1239-1247, 1996.

[11] E. M. Connor, R. S. Sperling, R. Gelber et al., "Reduction of maternal-infant transmission of human immunodeficiency virus type 1 with zidovudine treatment. Pediatric AIDS Clinical 
Trials Group Protocol 076 Study Group," The New England Journal of Medicine, vol. 331, no. 18, pp. 1173-1180, 1994.

[12] R. S. Sperling, D. E. Shapiro, G. D. McSherry et al., "Safety of the maternal-infant zidovudine regimen utilized in the Pediatric AIDS Clinical Trial Group 076 study," AIDS, vol. 12, no. 14, pp. 1805-1813, 1998.

[13] W. J. Watson, T. P. Stevens, and G. A. Weinberg, "Profound anemia in a newborn infant of a mother receiving antiretroviral therapy," Pediatric Infectious Disease Journal, vol. 17, no. 5, pp. 435-436, 1998.

[14] S. A. Myers, S. Torrente, D. Hinthorn, and P. L. Clark, "Lifethreatening maternal and fetal macrocytic anemia from antiretroviral therapy," Obstetrics and Gynecology, vol. 106, no. 5, part 2, pp. 1189-1191, 2005.

[15] M. Bunders, C. Thorne, and M. L. Newell, "Maternal and infant factors and lymphocyte, CD4 and CD8 cell counts in uninfected children of HIV-1-infected mothers," AIDS, vol. 19, no. 10, pp. 1071-1079, 2005.

[16] M. E. Paul, C. J. Chantry, J. S. Read et al., "Morbidity and mortality during the first two years of life among uninfected children born to human immunodeficiency virus type 1-infected women: the women and infants transmission study," Pediatric Infectious Disease Journal, vol. 24, no. 1, pp. 46-56, 2005.

[17] S. E. Pacheco, K. McIntosh, M. Lu et al., "Effect of perinatal antiretroviral drug exposure on hematologic values in HIVuninfected children: an analysis of the women and infants transmission study," Journal of Infectious Diseases, vol. 194, no. 8, pp. 1089-1097, 2006.

[18] M. Lallemant, G. Jourdain, S. Le Coeur et al., "A trial of shortened zidovudine regimens to prevent mother-to-child transmission of human immunodeficiency virus type 1," The New England Journal of Medicine, vol. 343, no. 14, pp. 982-991, 2000.

[19] J. S. Read, Y. Huo, K. Patel, M. Mitchell, and G. B. Scott, "Laboratory abnormalities among HIV-exposed, uninfected infants: IMPAACT protocol P1025," Journal of the Pediatric Infectious Diseases Society, vol. 1, no. 2, pp. 92-102, 2012.

[20] European Collaborative Study, "Levels and patterns of neutrophil cell counts over the first 8 years of life in children of HIV-1-infected mothers," AIDS, vol. 18, no. 15, pp. 2009-2017, 2004.

[21] L. Mandelbrot, A. Landreau-Mascaro, C. Rekacewicz et al., "Lamivudine-zidovudine combination for prevention of maternal-infant transmission of HIV-1," Journal of the American Medical Association, vol. 285, no. 16, pp. 2083-2093, 2001.

[22] J. M. Bellon Cano, S. Sanchez-Ramon, L. Ciria et al., “The effects on infants of potent antiretroviral therapy during pregnancy: a report from Spain," Medical Science Monitor, vol. 10, no. 5, pp. CR179-CR184, 2004.

[23] M. M. Mussi-Pinhata, M. A. C. Rego, L. Freimanis et al., "Maternal antiretrovirals and hepatic enzyme, hematologic abnormalities among human immunodeficiency virus type 1-uninfected infants: the NISDI perinatal study," Pediatric Infectious Disease Journal, vol. 26, no. 11, pp. 1032-1037, 2007.

[24] T. E. Taha, N. Kumwenda, A. Gibbons et al., "Effect of HIV-1 antiretroviral prophylaxis on hepatic and hematological parameters of African infants," AIDS, vol. 16, no. 6, pp. 851-858, 2002.

[25] L. Mandelbrot, F. Mazy, C. Floch-Tudal et al., "Atazanavir in pregnancy: impact on neonatal hyperbilirubinemia," European Journal of Obstetrics Gynecology and Reproductive Biology, vol. 157, no. 1, pp. 18-21, 2011.
[26] S. Dryden-Peterson, R. L. Shapiro, M. D. Hughes et al., "Increased risk of severe infant anemia after exposure to maternal HAART, Botswana," Journal of Acquired Immune Deficiency Syndromes, vol. 56, no. 5, pp. 428-436, 2011.

[27] C. Feiterna-Sperling, K. Weizsaecker, C. Bührer et al., "Hematologic effects of maternal antiretroviral therapy and transmission prophylaxis in HIV-1-exposed uninfected newborn infants," Journal of Acquired Immune Deficiency Syndromes, vol. 45, no. 1, pp. 43-51, 2007.

[28] J. Le Chenadec, M.-J. Mayaux, C. Guihenneuc-Jouyaux, and S. Blanche, "Perinatal antiretroviral treatment and hematopoiesis in HIV-uninfected infants," AIDS, vol. 17, no. 14, pp. 2053-2061, 2003.

[29] D. Nurutdinova, N. F. Onen, E. Hayes, K. Mondy, and E. T. Overton, "Adverse effects of tenofovir use in HIV-infected pregnant women and their infants," Annals of Pharmacotherapy, vol. 42, no. 11, pp. 1581-1585, 2008.

[30] S. Baroncelli, E. Tamburrini, M. Ravizza et al., "Antiretroviral treatment in pregnancy: a six-year perspective on recent trends in prescription patterns, viral load suppression, and pregnancy outcomes," AIDS Patient Care and STDs, vol. 23, no. 7, pp. 513520, 2009.

[31] R. Griner, P. L. Williams, J. S. Read et al., "In utero and postnatal exposure to antiretrovirals among hiv-exposed but uninfected children in the united states," AIDS Patient Care and STDs, vol. 25, no. 7, pp. 385-394, 2011.

[32] Division of AIDS and National Institutes of Health, Division of AIDS Table for Grading the Severity of Adult and Pediatric Adverse Events, Division of AIDS, National Institutes of Health, Bethesda, Md, USA, 2004, http://rsc.tech-res.com/ safetyandpharmacovigilance/gradingtables.aspx.

[33] A. Weinberg, J. Forster-Harwood, E. J. McFarland et al., "Resistance to antiretrovirals in HIV-infected pregnant women," Journal of Clinical Virology, vol. 45, no. 1, pp. 39-42, 2009.

[34] A. Weinberg, J. E. F. Harwood, E. J. McFarland et al., "Kinetics and determining factors of the virologic response to antiretrovirals during pregnancy," Infectious Diseases in Obstetrics and Gynecology, vol. 2009, Article ID 621780, 8 pages, 2009.

[35] A. Weinberg, J. Forster-Harwood, J. Davies et al., "Safety and tolerability of antiretrovirals during pregnancy," Infectious Diseases in Obstetrics and Gynecology, vol. 2011, Article ID 867674, 6 pages, 2011.

[36] C. Smith, J. E. Forster, M. J. Levin et al., "Serious adverse events are uncommon with combination neonatal antiretroviral prophylaxis: a retrospective case review," PLoS ONE, vol. 10, no. 5, Article ID e0127062, 2015.

[37] V. Venkatesh, R. Khan, A. Curley, H. New, and S. Stanworth, "How we decide when a neonate needs a transfusion," British Journal of Haematology, vol. 160, no. 4, pp. 421-433, 2013.

[38] S. A. McCormack and B. M. Best, "Protecting the fetus against HIV infection: a systematic review of placental transfer of antiretrovirals," Clinical Pharmacokinetics, vol. 53, no. 11, pp. 989-1004, 2014.

[39] A. Gingelmaier, M. Kurowski, R. Kästner et al., "Placental transfer and pharmacokinetics of lopinavir and other protease inhibitors in combination with nevirapine at delivery," AIDS, vol. 20, no. 13, pp. 1737-1743, 2006.

[40] C. Bennetto-Hood, Y. J. Bryson, A. Stek, J. King, M. Mirochnick, and E. Acosta, "Zidovudine, lamivudine, and nelfinavir concentrations in amniotic fluid and maternal serum," HIV Clinical Trials, vol. 10, no. 1, pp. 41-47, 2009. 
[41] A. Colbers, R. Greupink, and D. Burger, "Pharmacological considerations on the use of antiretrovirals in pregnancy," Current Opinion in Infectious Diseases, vol. 26, no. 6, pp. 575-588, 2013.

[42] Panel on Treatment of HIV-Infected Pregnant Women and Prevention of Perinatal Transmission, Recommendations for Use of Antiretroviral Drugs in Pregnant HIV-1-Infected Women for Maternal Health and Interventions to Reduce Perinatal HIV Transmission in the United States, 2015.

[43] T. Eley, S.-P. Huang, F. Conradie et al., "Clinical and pharmacogenetic factors affecting neonatal bilirubinemia following atazanavir treatment of mothers during pregnancy," AIDS Research and Human Retroviruses, vol. 29, no. 10, pp. 1287-1292, 2013.

[44] A. Vivanti, T. S. Soheili, W. Cuccuini et al., "Comparing genotoxic signatures in cord blood cells from neonates exposed in utero to zidovudine or tenofovir," AIDS, vol. 29, no. 11, pp. 13191324, 2015.

[45] M. Perry, G. P. Taylor, C. A. Sabin et al., "Lopinavir and atazanavir in pregnancy: comparable infant outcomes, virological efficacies and preterm delivery rates," HIV Medicine, vol. 17, no. 1, pp. 28-35, 2016. 


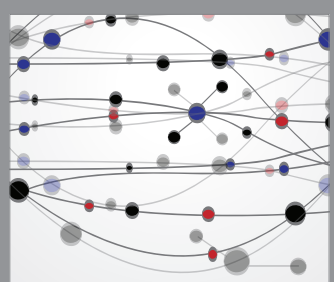

The Scientific World Journal
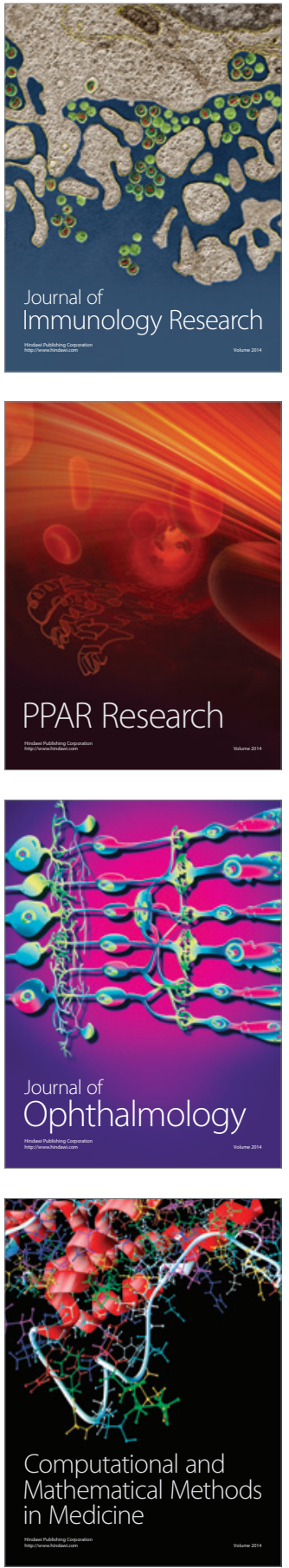

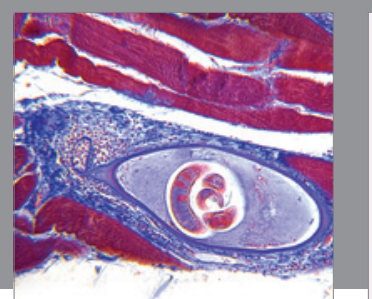

Gastroenterology Research and Practice

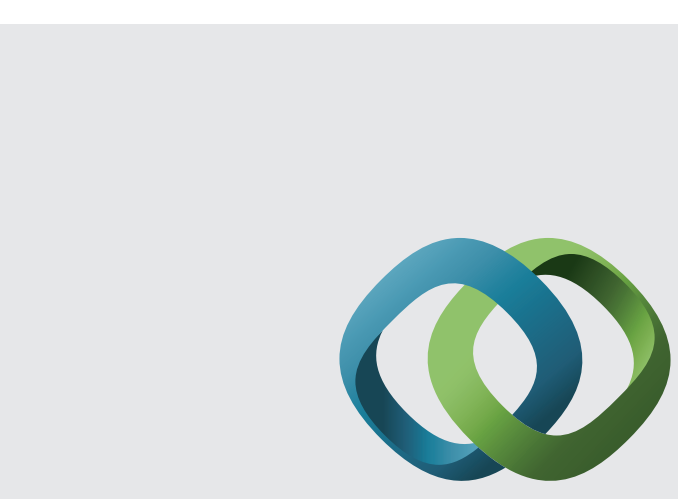

\section{Hindawi}

Submit your manuscripts at

http://www.hindawi.com
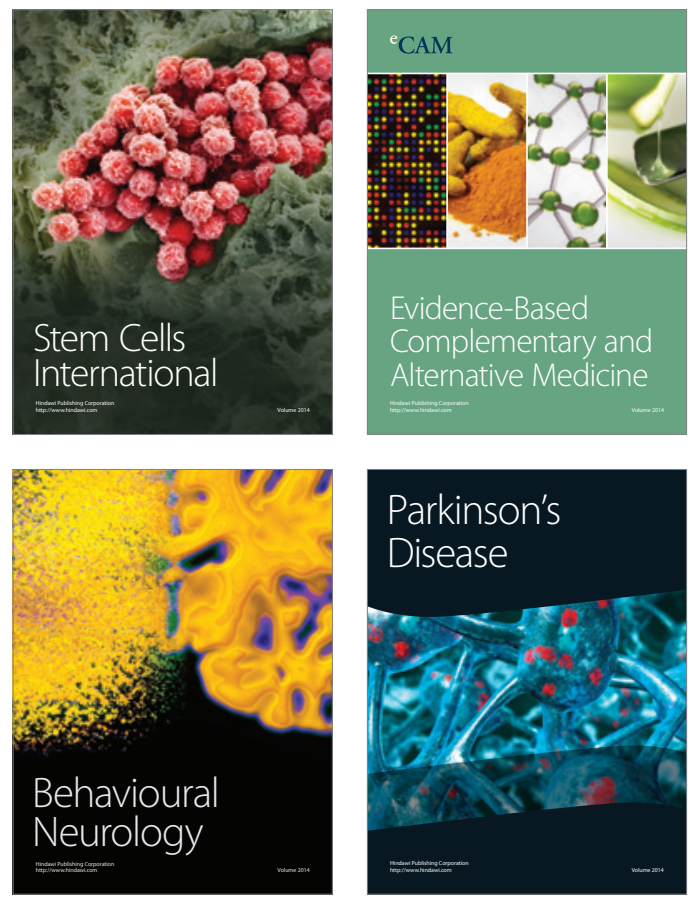
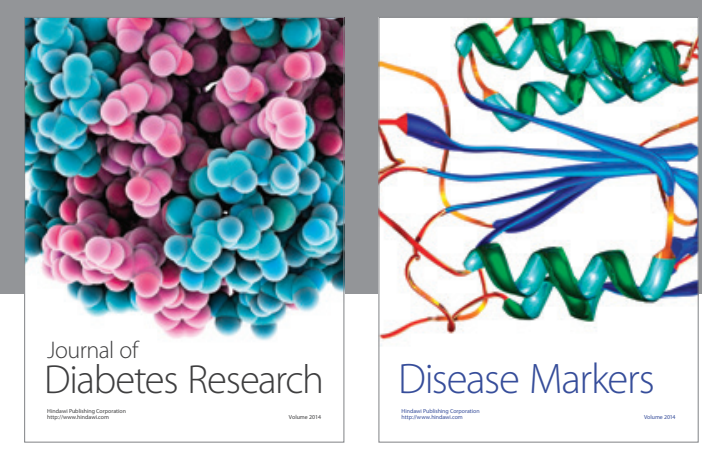

Disease Markers
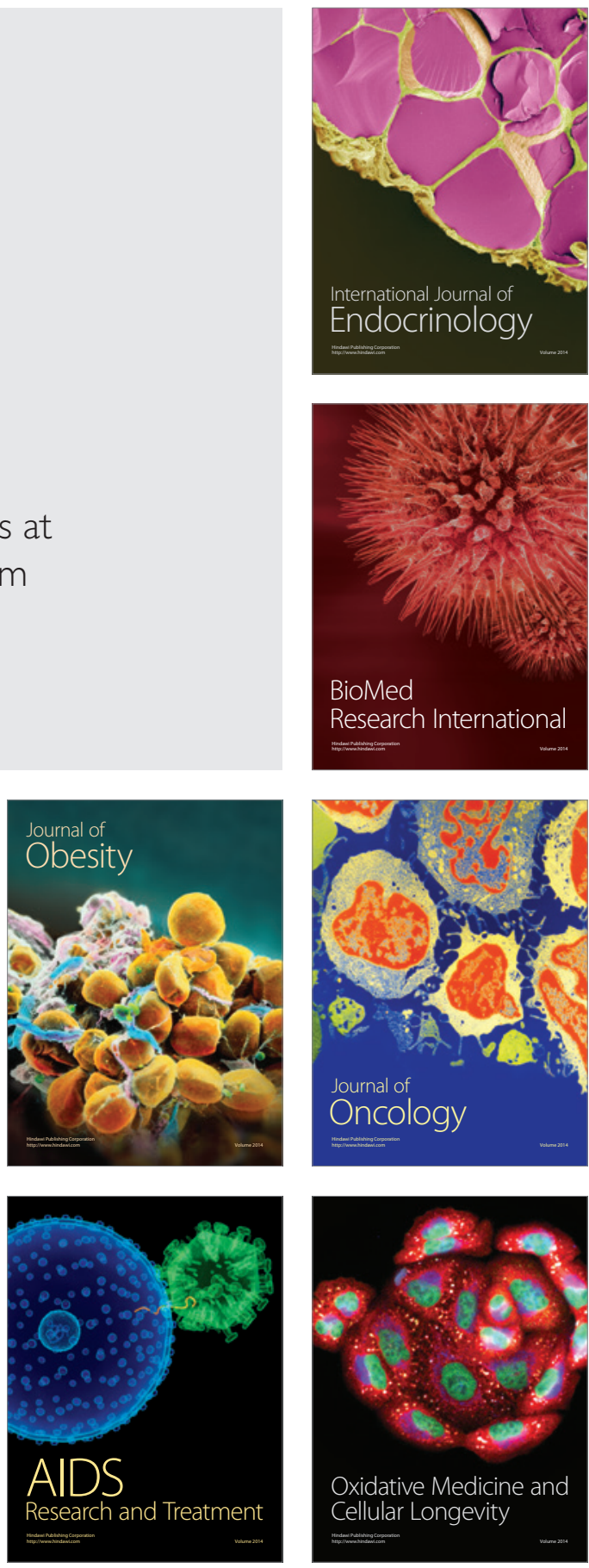